Proceedings of the 9th National Symposium of Synchrotron Radiation Users, Warsaw, September 26-27, 2011

\title{
Synchrotron Topographic Studies of Domain Structure in Czochralski Grown $\mathrm{Pr}_{x} \mathrm{La}_{1-x} \mathrm{AlO}_{3}$ and $\mathrm{Pr}_{x} \mathrm{La}_{1-x-y} \mathrm{Mg}_{y} \mathrm{AlO}_{3}$ Crystals
}

\author{
K. Wieteska ${ }^{a}$, W. Wierzchowski ${ }^{b}$, A. Malinowska ${ }^{b}$, S. TurCZyŃSki $^{b}$, \\ M. Lefeld-Sosnowska ${ }^{c}$, D.A. PaWlak ${ }^{b}$, T. Eukasiewicz ${ }^{b}$ And C. Paulmann ${ }^{d}$ \\ ${ }^{a}$ National Centre for Nuclear Research, A. Soltana 7, Otwock-Świerk 05-400, Poland \\ ${ }^{b}$ Institute of Electronic Materials Technology, Wólczyńska 133, Warszawa 01-919, Poland \\ ${ }^{c}$ Warsaw University, Institute of Experimental Physics, Hoża 69, Warszawa 00-681, Poland \\ ${ }^{d}$ HASYLAB at DESY, Notkestrasse 85, D-22607 Hamburg, Germany
}

\begin{abstract}
A domain structure and crystallographic defects in Czochralski grown single crystals of $\operatorname{Pr}_{x} \operatorname{La}_{1-x} \mathrm{AlO}_{3}$ and $\operatorname{Pr}_{x} \mathrm{La}_{1-x-y} \mathrm{Mg}_{y} \mathrm{AlO}_{3}$ were characterised with a number of methods including conventional and synchrotron X-ray diffraction topography, and polariscopic micrography. The observed twin domain systems were located perpendicularly to $\langle 100\rangle_{\text {pcub }}$ and $\langle 110\rangle_{\text {pcub }}$ (pseudocubic) directions. It has been confirmed that the domains are of the same orientation and a twin character as those described in literature for $\mathrm{LaAlO}_{3}$ and $\mathrm{LaGaO}_{3}$ crystals. The use of section transmission topography enabled to indicate that the domains are perpendicular to the $(100)_{\text {pcub }}$ surface of the samples. The misorientation of lattice in the domains was evaluated from the white beam topographs and a tendency of its increase with increasing concentration of praseodymium was revealed.
\end{abstract}

PACS: 81.05.-t, 81.10.Dn, 61.72.Ff

\section{Introduction}

The technology of growth of mixed praseodymium lanthanum aluminate perovskite crystals is developed mainly for some perspective applications in visible light laser technology. One of the aspects of the interest is connected with the preparation of crystals with selforganized domain structure with possible application in light guiding [1].

The structure of $\mathrm{PrAlO}_{3}$ and $\mathrm{LaAlO}_{3}$ has been studied by Glazer [2, 3], Megaw and Darlington [4], and by Zhao et al. [5]. At room temperature, these compounds adopt the structure of rhombohedrally distorted perovskite (space group $R \overline{3} c$, tilt system $a^{-} a^{-} a^{-}$). Lattice parameters given in hexagonal notation are: $a_{h}=$ $5.3328 \AA, c_{h}=12.973 \AA$ for $\mathrm{PrAlO}_{3}$ crystal [1] and $a_{h}=5.3653 \AA, c_{h}=13.112 \AA$ for $\mathrm{LaAlO}_{3}$ [6]. It should be noted, however, that small discrepancies in the lattice parameters reported by several authors were observed and can be caused by twinning structure, which is generated in the $\mathrm{PrAlO}_{3}$ and $\mathrm{LaAlO}_{3}$ rhombohedral phases. Some physical properties of these crystals were described in [7-14]. The crystals are very interesting in view of the complicated phase transitions. At $833 \mathrm{~K}$ $\mathrm{LaAlO}_{3}$ crystal exhibits a phase transition from lowtemperature rhombohedral phase to high-temperature cubic phase [12]. $\mathrm{PrAlO}_{3}$ differs from $\mathrm{LaAlO}_{3}$ in the value of this phase transition temperature and undergoes further phase transitions below room temperature: $C 2 / m \stackrel{150 K}{\longrightarrow} I m m a \stackrel{205 K}{\longrightarrow} R \overline{3} c \stackrel{1770 K}{\longrightarrow} \operatorname{Pm} \overline{3} m$ [8]. The phase transitions in $\mathrm{PrAlO}_{3}$ crystal were also recently studied by Carpenter et al. [14] providing in particular the corrected value of $R \overline{3} c \longrightarrow P m \overline{3} m$ transition temperature as $1864( \pm 31) \mathrm{K}$.

$\mathrm{Pr}_{x} \mathrm{La}_{1-x} \mathrm{AlO}_{3}$ solid solution perovskites are very interesting in view of their many phase transitions (which can be engineered by changing the Pr:La ratio) and the change of electronic, magnetic and structural properties [15-17].

The important problem in the technology of rare earth aluminates crystals is the characterisation including examination of the regularity and crystallographic features of the domain structure, and the presence of common crystallographic defects, as dislocations and inclusions. The properties and the defect structure of such crystals grown in the Institute of Electronic Materials Technology were published elsewhere $[1,5,9-11,13-18]$. In particular, the powder X-ray diffractometric results confirmed the suggested rhombohedral crystallographic structure of the crystals.

An important aspect of the interest is connected with domain structure which is generated in the $\mathrm{Pr}_{x} \mathrm{La}_{1-x} \mathrm{AlO}_{3}$ rhombohedral phases. It has been known that X-ray diffraction topograph methods are very useful tools for twin structure studies [18-26]. The domain structure in $\mathrm{LaAlO}_{3}$ crystals has been studied with X-ray diffraction topography by Dudley and Yao [24-25], especially as a function of temperature in the vicinity of phase transition temperatures [25]. These authors have found, in particular, that in $\mathrm{LaAlO}_{3}$, which at room temperature has a similar $R \overline{3} c$ structure, the domains are located along the $\{110\}_{\text {rhom }}$ and $\{211\}_{\text {rhom }}$ planes, which corre- 
spond to $\{100\}_{\text {pcub }}$ and $\{110\}_{\text {pcub }}$ planes in the pseudocubic lattice, respectively.

In the present paper we studied the domain and defect structure of Czochralski-grown $\mathrm{Pr}_{x} \mathrm{La}_{1-x} \mathrm{AlO}_{3}$ and $\mathrm{Pr}_{x} \mathrm{La}_{1-x-y} \mathrm{Mg}_{y} \mathrm{AlO}_{3}$ with a wider set of chemical composition. The single crystals were characterised with a number of methods including conventional and synchrotron X-ray diffraction topography, and polariscopic micrography. The presently applied sample orientation allowed precise recognition of domain systems, and determination of the orientation of the lattice connected with the domains.

\section{Experimental}

The examined crystals were grown by the Czochralski method [1]. High purity $\mathrm{Pr}_{6} \mathrm{O}_{11}, \mathrm{Al}_{2} \mathrm{O}_{3}$ and $\mathrm{La}_{2} \mathrm{O}_{3}$ oxides $(99.995 \%)$ were used as raw materials. All components were mixed in stoichiometric ratios. The pulling and rotation rates were in the range of $1-1.7 \mathrm{~mm} / \mathrm{h}$ and $6-8 \mathrm{rpm}$. The crystals were grown in pure nitrogen atmosphere. The samples were cut out perpendicularly to the $\langle 100\rangle_{\text {pcub }}$ pseudo-cubic axis closest to the actual growth direction.

The presently investigated crystals included $\mathrm{Pr}_{x} \mathrm{La}_{1-x} \mathrm{AlO}_{3}$ with $x$ equal to: $1,0.75,0.55,0.4$, $0.05,0.03,0.01$ and $0 . \operatorname{Pr}_{x} \mathrm{La}_{1-x-y} \mathrm{Mg}_{y} \mathrm{AlO}_{3}$ crystals with $x=0.042$ and $y=0.021, x=0.034$ and $y=0.017$, $x=0.028$ and $y=0.028, x=0.023$ and $y=0.023$ were also studied. The samples were mechanically and chemically polished and studied with a number of $\mathrm{X}$-ray diffraction topographic methods exploring both synchrotron and conventional sources.

The synchrotron X-ray topographic experiments were performed using a white beam in back-reflection or transmission geometry. The conventional X-ray Lang topographs were taken in back-reflection geometry for selected samples. The transmission section topographs exposed with the beam perpendicular to the crystal surface, which allowed easy recognition of the domains perpendicular to the surface, were very important for the identification of the geometric features of the domain structure.

The details of the domain structure were also well revealed in polariscopic micrographs. A certain surface relief corresponding to the domains was also observed by means of the precise profilometer. This surface relief is most probably caused by different mechanical or chemical polishing of differently oriented materials inside the domains.

\section{Results and discussion}

Some of presently investigated as-grown crystals are shown in Fig. 1. The crystals differ in colour, and, in some cases, some irregularities of their form were observed. One can notice a certain deformation of the crystal rod and subsequent tendency of spiral growth in case of materials with high praseodymium concentration, see
$\operatorname{Pr}_{0.4} \mathrm{La}_{0.6} \mathrm{AlO}_{3}$ crystal shown in Fig. 1a. In view of the further results the deformation is most probably caused by the phase transition inside the crystals.

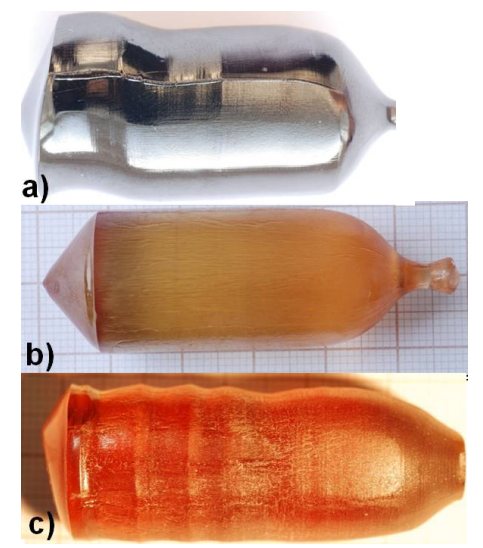

Fig. 1. The photographs of the representative as grown crystals: (a) $\mathrm{Pr}_{0.4} \mathrm{La}_{0.6} \mathrm{AlO}_{3}$ (b) $\mathrm{Pr}_{0.01} \mathrm{La}_{0.99} \mathrm{AlO}_{3}$ and (c) $\mathrm{Pr}_{0.042} \mathrm{La}_{0.937} \mathrm{Mg}_{0.021} \mathrm{AlO}_{3}$

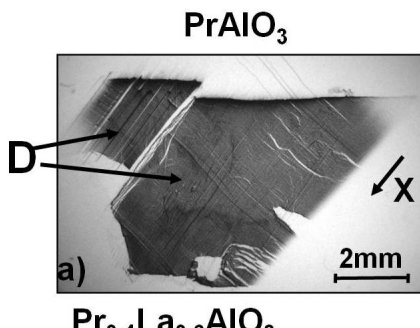

$\operatorname{Pr}_{0.75} \mathrm{La}_{0.25} \mathrm{AlO}$

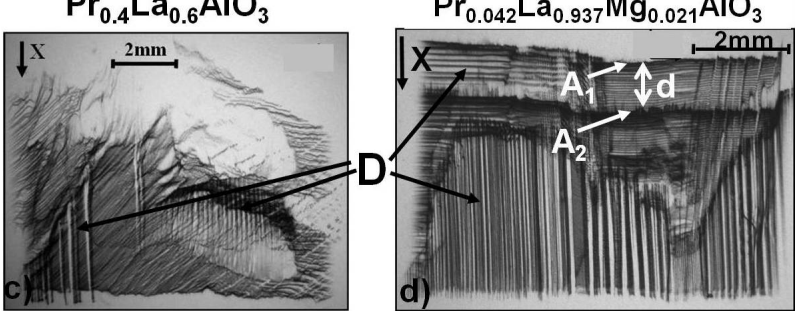

Fig. 2. White beam projection back-reflection diffraction topographs of the samples with various chemical composition. X denotes projection of the synchrotron radiation beam direction on the film; $\mathrm{D}$ - sets of domains: $A_{1}, A_{2}$ - mutually displaced images of boundaries of different domains sets present in the same illuminated sample region.

Representative white beam synchrotron back-reflection projection topographs of the samples with various chemical compositions are shown in Figs. 2, 3 and 4 . In all these topographs one can notice several systems of mutually displaced stripes, each of them revealing different region of the crystal corresponding to the domain structure. The appearance of the stripes is different in the samples with different chemical composition. They tend to be less numerous and widely spaced in the samples with low concentration of praseodymium and most distinct in the crystals containing both praseodymium and magnesium. In some of the samples with relatively 

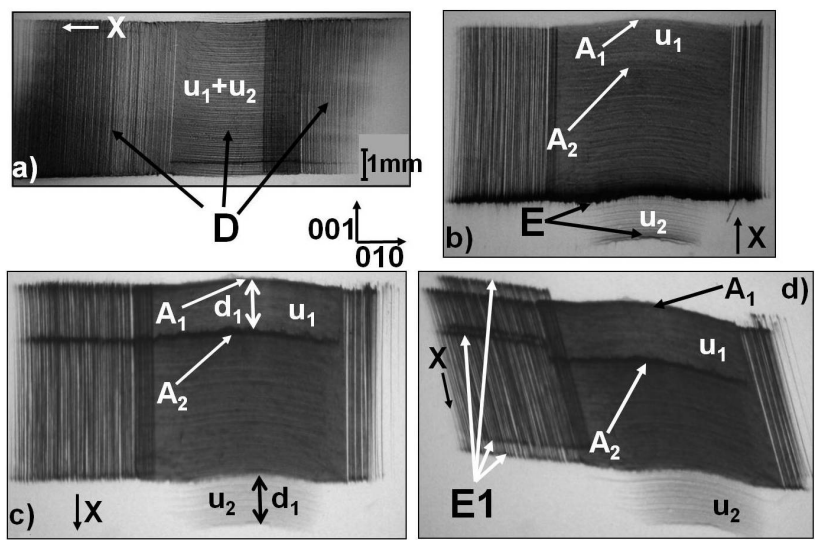

Fig. 3. (a)-(c) White beam projection back-reflection diffraction topographs taken at three azimuths of $\mathrm{Pr}_{0.05} \mathrm{La}_{0.95} \mathrm{AlO}_{3}$ in reflection from equivalent $\{310\}$ planes after successive rotation through $90^{\circ}$; (d) One of the skew reflections for the same azimuth as (b). $\mathrm{u}_{1}, \mathrm{u}_{2}$ - images of various sets of domains present in the same crystal region; $A_{1}, A_{2}$ - boundaries of images of $\mathbf{u}_{1}$ and $\mathrm{u}_{2} ; \mathrm{d}_{1}$ - the image displacement; $\mathrm{X}$ denotes projection of the synchrotron radiation beam direction on the film; $\mathrm{D}$ - sets of domains; $\mathrm{E}$ - displaced images of domains $\mathrm{u}_{1}$ and $\mathbf{u}_{2}$ present in the same illuminated sample region; E1 - additional displacement of domains images not seen in parts (b) and (c).

dense stripes, complementary images of the neighbouring stripes are significantly displaced and one can have the impression of the double image of the whole sample as may be seen e.g. in Fig. $2 \mathrm{~d}$ and 5 in the upper part of the topographs. The mutually displaced images correspond to the disoriented domains. In white beam synchrotron radiation back-reflection topography the domains may choose the appropriate wavelength meeting the Bragg condition, which results in the similar diffracted beam intensity but reflected along slightly different directions and gives mutually displaced images.

One can notice that a majority of stripes are straight and they are located in two perpendicular $\langle 100\rangle_{\text {pcub }}$ directions. A smaller number of stripes is located along $\langle 110\rangle_{\text {pcub }}$ directions and a small part exhibits a certain curvature. It may be expected that the curvature corresponds to some kinks, which were revealed in Nomarski topographs reported in [1].

Figs. 3a, 3b, 3c represent the topographs taken for a $\operatorname{Pr}_{0.05} \mathrm{La}_{0.95} \mathrm{AlO}_{3}$ crystal in pseudocubic 226 reflections from equivalent crystallographic planes for three azimuths differing through $90^{\circ}$. The different directions of the primary SR beam are marked as $\mathrm{X}$ in the figures. In the topographs one can recognize three different regions with different sets of stripes. At least two sets of domains are present in each region (e.g. $\mathrm{u}_{1}$ and $\mathrm{u}_{2}$ in the middle one). They indicate that the two systems of domains (forming weaker and stronger images) correspond to a small change of orientation in the plane perpendicular to the domains, expected to the twin character of the domains. The diffraction images of these domains are most
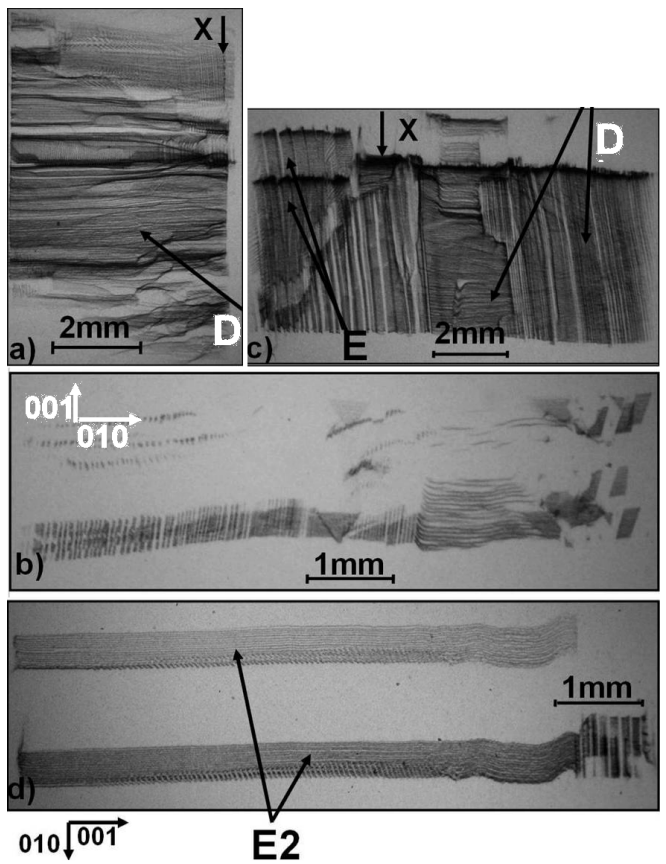

Fig. 4. (a) (c) - white beam projection back-reflection diffraction topographs of $\operatorname{Pr}_{0.034} \mathrm{La}_{0.949} \mathrm{Mg}_{0.017} \mathrm{AlO}_{3}$ in two azimuths differing through $90^{\circ}$; (b) (d) - white beam transmission section diffraction topographs in the same two azimuths (as in topographs (a) and (b)) differing through $90^{\circ}$; Displaced fragments of the image of the slit correspond to the differently oriented crystal blocks and sets of domains; The vertical location of some domain contrasts in the white beam transmission section topographs proves their perpendicularity to the surface of the samples; $X$ denotes projection of the synchrotron radiation beam direction on the film; D - sets of domains; $\mathrm{E}$ - displaced images of different domains present in the same illuminated sample region; E2 displaced fragments of the image of the slit corresponding to the different sets of domains.

distinctly displaced in situation when the stripes are perpendicular to the SR beam, when the misorientation is in the diffraction plane. Contrary to that, the displacement becomes almost negligible when the domains are parallel to the plane of diffraction. We may also notice that the representative skew reflection, shown in Fig. 3d reveal also a certain displacement of the images of the domains parallel to the direction of the beam.

The other proof indicating the twin character of the domains comes from the whole synchrotron Laue pattern shown in Fig. 5, where the displaced systems of domains are similar in each Laue spot and the difference of the displacement is only due to different projection of the diffracted beam.

The appearance of the stripes seems to be in a very good agreement with the results of Dudley and Yao [25] that the domains are located along the $\{110\}_{\text {rhom }}$ and $\{211\}_{\text {rhom }}$ planes, which correspond to $\{100\}_{\text {pcub }}$ and $\{110\}_{\text {pcub }}$ planes in the pseudocubic lattice, respectively. 


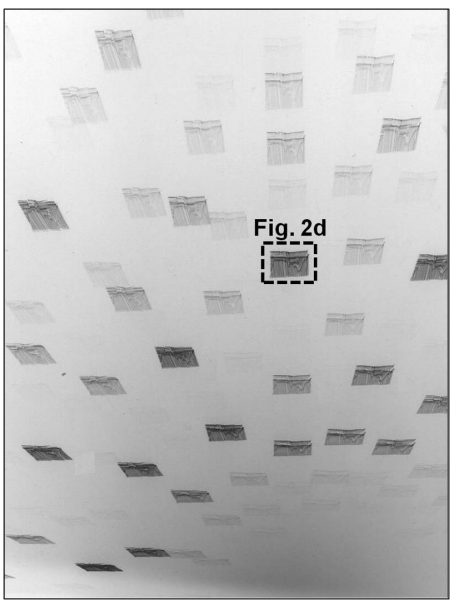

Fig. 5. Example of full white beam synchrotron pattern confirming of the twin character of the domains. One can notice that the images of all different domains are present in the vicinity of each Laue spot. The marked region corresponds to the topograph in Fig. 2d.
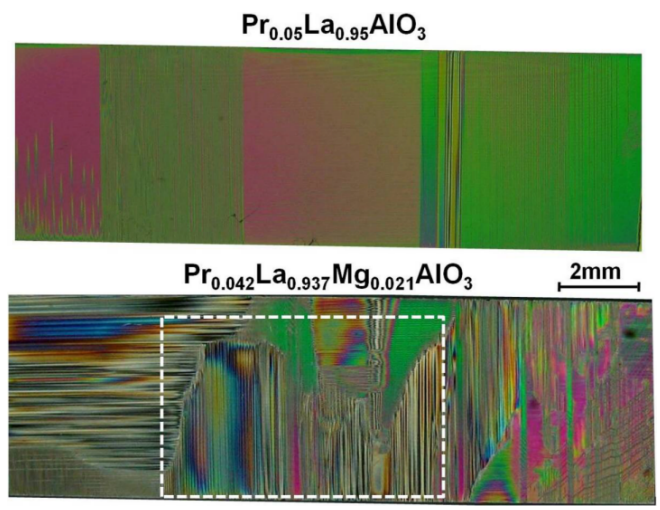

Fig. 6. Polariscopic micrographs of two selected samples with various compositon: (a) $\operatorname{Pr}_{0.05} \mathrm{La}_{0.95} \mathrm{AlO}_{3}$ crystal; micrograph of the same region as Fig. 3a,b $\mathrm{Pr}_{0.042} \mathrm{La}_{0.937} \mathrm{Mg}_{0.021} \mathrm{AlO}_{3}$ crystal; the marked rectangle corresponds to the topograph in Fig. 2d.

A very important proof for that was obtained from the section topographs shown representatively in Fig. $4 \mathrm{~b}$ and 4d. As these topographs were exposed with the beam perpendicular to the surface, the observed vertical images of domains must correspond to the domains perpendicular to the surface.

As one can see in Fig. 6, the polariscopic micrographs can also reveal the domain structure present in the investigated materials. Contrary to the X-ray topography, the contrast may be attributed to the stress connected with the domains and other crystallographic defects, while the effect of the lattice misorientation is not revealed. That is why the polariscopic images are often more clear, although the possibility of revealing the individual crystallographic defects, e.g.dislocation is usually much worse than in the case of X-ray diffraction topography.

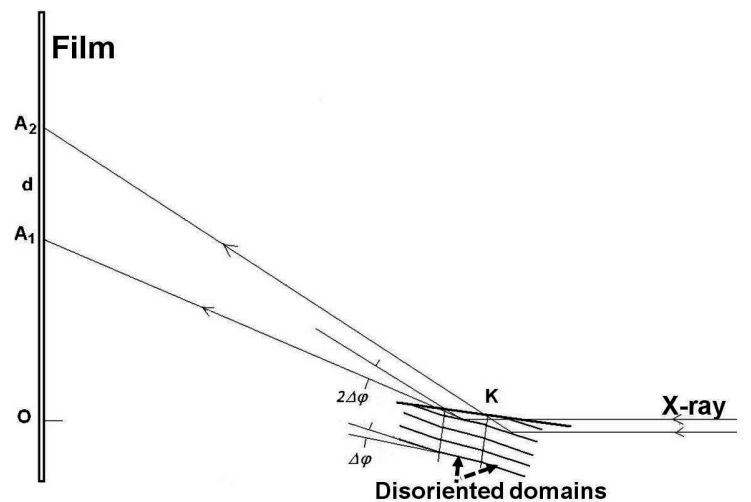

Fig. 7. Schema of the evaluation of the lattice misorientation between domains. The width of the domains and the misorientation of the lattice is exaggerated. The distance $\mathrm{A}_{1} \mathrm{~A}_{2}$ is representatively marked in Fig. 2d (as d) and $3 c\left(\operatorname{as~} d_{1}\right)$.

TABLE

The lattice misorientation between representative $\{110\}$ type system of domains evaluated from the image displacement in white beam projection topographs.

\begin{tabular}{lcc}
\hline \hline & Chemical composition & Evaluated misorientation \\
\hline 1 & $\mathrm{LaAlO}_{3}$ & $0.12^{\circ} \pm 0.01^{\circ}$ \\
\hline 2 & $\mathrm{Pr}_{0.01} \mathrm{La}_{0.99} \mathrm{AlO}_{3}$ & $0.2^{\circ} \pm 0.01^{\circ}$ \\
\hline 3 & $\mathrm{Pr}_{0.023} \mathrm{La}_{0.954} \mathrm{Mg}_{0.023} \mathrm{AlO}_{3}$ & $0.14^{\circ} \pm 0.01^{\circ}$ \\
\hline 4 & $\mathrm{Pr}_{0.028} \mathrm{La}_{0.944} \mathrm{Mg}_{0.028} \mathrm{AlO}_{3}$ & $0.24^{\circ} \pm 0.01^{\circ}$ \\
\hline 5 & $\mathrm{Pr}_{0.03} \mathrm{La}_{0.97} \mathrm{AlO}_{3}$ & $0.30^{\circ} \pm 0.01^{\circ}$ \\
\hline 6 & $\mathrm{Pr}_{0.034} \mathrm{La}_{0.949} \mathrm{Mg}_{0.017} \mathrm{AlO}_{3}$ & $0.28^{\circ} \pm 0.01^{\circ}$ \\
\hline 7 & $\operatorname{Pr}_{0.042} \mathrm{La}_{0.937} \mathrm{Mg}_{0.021} \mathrm{AlO}_{3}$ & $0.32^{\circ} \pm 0.01^{\circ}$ \\
\hline 8 & $\operatorname{Pr}_{0.4} \mathrm{La}_{0.6} \mathrm{AlO}_{3}$ & $0.28^{\circ} \pm 0.01^{\circ}$ \\
\hline 9 & $\operatorname{Pr}_{0.55} \mathrm{La}_{0.45} \mathrm{AlO}_{3}$ & $0.30^{\circ} \pm 0.01^{\circ}$ \\
\hline 10 & $\operatorname{Pr}_{0.75} \mathrm{La}_{0.25} \mathrm{AlO}_{3}$ & $0.6^{\circ} \pm 0.01^{\circ}$ \\
\hline 11 & $\mathrm{PrAlO}_{3}$ & $1^{\circ} \pm 0.01^{\circ}$
\end{tabular}

It was possible to compare the misorientation between $\{110\}_{\text {rhomb }}$ domains for the samples with different chemical composition. In order to do that, the topographs for the same orientation (with the projection of the incident beam along $\langle 100\rangle$ ), were taken for all investigated samples. Although the topographs revealed many domain systems usually in each particular system the same misorientation of domains was observed. In particular for chosen domain systems along $\{110\}_{\text {rhomb }}$ the topographs corresponding to the zero-layer reflections revealed misorientations with the only component in diffraction plane. The misorientation is relatively large and may be evaluated with a good accuracy.

The misorientation was evaluated from the displacement of the images corresponding to the two different sets of domains topographs corresponding to mixed 620 and 310 pseudocubic reflections. The schema of the method is shown in Fig. 7. It may be easily noticed that the double value of the Bragg angle is approximately equal to: 


$$
2 \Theta=\arctan \left(\left|O A_{1}\right| /|O K|\right)
$$

hence

$$
\begin{aligned}
\Delta \phi & =d \cos (2 \Theta) /(|O K| / \cos (2 \Theta)) \\
\Delta \phi & =d \cos ^{2}(2 \Theta) / 2(|O K|) .
\end{aligned}
$$

The above formula uses the arc angles, however, it is more useful to give the values of misorientation in degrees $\left(1^{\circ}=\pi / 180 \mathrm{rad}\right)$.

The uncertainty of evaluated misorientation angles is of the order of $\pm 0.01^{\circ}$ and comes from uncertainties of measured distances $\left|O A_{1}\right|,|O K|\left(\triangle\left|O A_{1}\right|=\right.$ $\Delta|O K|= \pm 1 \mathrm{~mm})$ and of displacements of images $d$ $(\Delta d= \pm 0.1 \mathrm{~mm})$. The values of misorientation are listed in Table. One can notice a tendency of the evaluated misorientation to increase (up to $1^{\circ}$ ) with an increase of praseodymium concentration for large values of $x(0.4 \leq x \leq 1)$. However some spread of misorientation in the range of $\left\langle 0.12^{\circ} ; 0.32^{\circ}\right\rangle$ for small values of $x(x \leq 0.042)$ was observed. Detailed determination of this dependence requires further investigations for whole range of praseodymium concentration.

\section{Conclusions}

The domain systems, observed by us, were investigated in $\mathrm{Pr}_{x} \mathrm{La}_{1-x} \mathrm{AlO}_{3}$ and $\mathrm{Pr}_{x} \mathrm{La}_{1-x-y} \mathrm{Mg}_{y} \mathrm{AlO}_{3}$ crystals, and their appearance and configuration were compared for samples, containing different amount of praseodymium. It has been confirmed that the domains are of a twin character, similarly as it was observed in $\mathrm{LaAlO}_{3}$ and $\mathrm{LaGaO}_{3}$ crystals and described in literature.

The observed twin domains systems were located in the $\{110\}_{\text {rhom }}$ and $\{211\}_{\text {rhom }}$ planes, which correspond to $\{100\}_{\text {pcub }}$ and $\{110\}_{\text {pcub }}$ planes in the pseudo-cubic lattice, respectively. The important proof that the domain systems are perpendicular to the (100) sample surface was obtained from the white beam transmission section topographs, which were exposed with the beam perpendicular to the sample surface.

The evaluated changes of the lattice orientation depend on the chemical composition of the samples, showing a tendency of increasing up to $1^{\circ}$ with increasing concentration of praseodymium for large values of $x(0.4 \leq x \leq$ 1). This tendency seems to correspond with the increasing density and lowering thickness of domains. For small values of $x(x \leq 0.042)$ some spread of misorientation in the range of $\left\langle 0.12^{\circ} ; 0.32^{\circ}\right\rangle$ was observed. Detailed determination of this dependence requires further investigations for whole range of praseodymium concentration.

\section{Acknowledgements}

The synchrotron investigations were supported by the EC 20100001 HASYLAB project. The authors also thank the IUVENTUS PLUS 2010 Project (IP2010044070).

\section{References}

[1] D.A. Pawlak, T. Łukasiewicz, M. Carpenter, M. Malinowski, R. Diduszko, J. Kisielewski, J. Cryst. Growth 282, 260 (2005).

[2] A.M. Glazer, Acta Crystallogr. B 28, 3384 (1972).
[3] A.M. Glazer, Acta Crystallogr. A 31, 756 (1975).

[4] H.D. Megaw, C.N.W. Darlington, Acta Crystallogr. A 31, 161 (1975).

[5] J. Zhao, N. L. Ross, R. J. Angel, M. A. Carpenter, Ch. J. Howard, D.A. Pawlak, T. Łukasiewicz, J. Phys.: Condens. Mat. 21, 235403 (2009).

[6] H. Lehnert, H. Boysen, P. Dreier, Y. Yu, Z. Kristallogr. 215, 145 (2000).

[7] C.J. Howard, B.J. Kennedy, B.C. Chakoumakos, J. Phys.: Condens. Mat. 12, 349 (2000).

[8] S.M. Moussa, B.J. Kennedy, B.A. Hunter, C.J. Howard, T. Voigt, J. Phys.: Condens. Mat. 13, L203 (2001).

[9] C. Filipič, V. Bobnar, S. Turczyński, D.A. Pawlak, M. Wencka, J. Dolinšek, A. Levstik, J. Appl. Phys. 108, 116102 (2010).

[10] M. Wencka, S. Vrtnik, M. Jagodič, Z. Jagličić, S. Turczynski, D.A. Pawlak, J. Dolinšek, Phys. Rev. B 80, 224410 (2009).

[11] M. Malinowski, M. Kaczkan, S. Turczyński, D.A. Pawlak, Opt. Mater. 33, 1004 (2011).

[12] M.M.C. Chou, Ch. Chen, S.S. Yang, Ch.H. Huang, H.L. Huang, J. Phys. Chem. Solids 69, 425 (2008).

[13] M. Kruczek, E. Talik, D.A. Pawlak, K. Kołodziejak, T. Łukasiewicz, J. Alloy. Compd. 442, 255 (2007).

[14] M.A. Carpenter, E.C. Wiltshire, C.J. Howard, R.I. Thomson, S. Turczynski, D.A. Pawlak, T. Łukasiewicz, Phase Transit. 83, 703 (2010).

[15] M. Szubka, E. Talik, A. Molak, S. Turczyński, D.A. Pawlak, Cryst. Res. Technol. 45, 1309 (2010).

[16] R.I. Thomson, J.M. Rawson, C.J. Howard, S. Turczynski, D.A. Pawlak, T. Łukasiewicz, M.A. Carpenter, Phys. Rev. B 82, 214111 (2010).

[17] S. Turczynski, K. Orliński, D.A. Pawlak, R. Diduszko, J. Mucha, M. Pekala, J.F. Fagnard, Ph. Vanderbemden, M.A. Carpenter, Cryst. Growth Des. 11, 1091 (2011).

[18] K. Wieteska, W. Wierzchowski, A. Malinowska, S. Turczyński, M. Lefeld-Sosnowska, D.A. Pawlak, T. Łukasiewicz and W. Graeff, Acta Phys. Pol. A 117, 268 (2010).

[19] G.-D. Yao, M. Dudley, Y. Wang, X. Liu, R.C. Liebermann, Mater. Sci. Eng. A 132, 23 (1991).

[20] J. Fink-Finowicki, M. Berkowski, A. Pajączkowska, J. Mater. Sci. 27, 107 (1992).

[21] K. Mazur, J. Fink-Finowicki, M. Berkowski, Acta Phys. Pol. A 92, 205 (1997).

[22] I.K. Bdikin, I.M. Shmyt'ko, A.M. Balbashov, A.V. Kazansky, J. Appl. Crystallogr. 26, 71 (1993).

[23] D. Savytskii, A. Senyshyn, A. Matkovskii, L. Vasylechko, K. Wieteska, W. Wierzchowski, T. Łukasiewicz, U. Bismayer, Z. Kristallogr. 218, 17 (2003).

[24] G.-D. Yao, S.Y. Hou, M. Dudley, J.M. Phillips, J. Mater. Res. 7, 1847 (1992).

[25] M. Dudley, G.-D. Yao, J. Phys. D 26, A120 (1993).

[26] M. G. Norton, R. R. Biggers, Scripta Metall. Mater. 32, 481 (1995). 